\title{
Metal bioremediation by CrMTP4 over-expressing Chlamydomonas reinhardtii in comparison to natural wastewater-tolerant microalgae strains
}

Aniefon Ibuot ${ }^{\mathrm{a}, \#}$, Andrew P. Dean ${ }^{\mathrm{b}}$, Owen Mclntosh ${ }^{\mathrm{c}}$, and Jon K. Pittman ${ }^{\mathrm{a}, \mathrm{c}, *}$

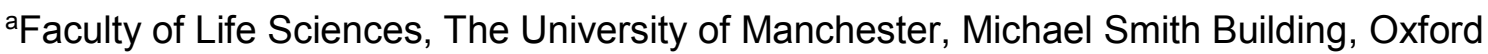
Road, Manchester M13 9PT, UK; 'bchool of Science and the Environment, Faculty of Science and Engineering, Manchester Metropolitan University, Chester Street, Manchester,

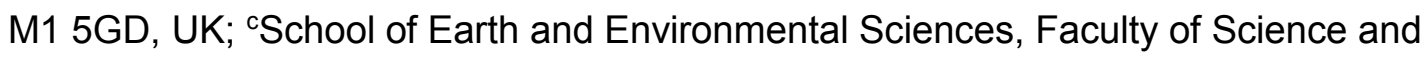
Engineering, The University of Manchester, Michael Smith Building, Oxford Road, Manchester M13 9PT, UK

${ }^{*}$ Corresponding Author:

Dr Jon Pittman, School of Earth and Environmental Sciences, University of Manchester, Michael Smith Building, Oxford Road, Manchester M13 9PT, UK; Tel: +44 (0)161 275 5235; Fax: +44 (0)161 275 5082; Email: jon.pittman@manchester.ac.uk

\#Present address: Department of Science Technology, Akwa Ibom State Polytechnic, Ikot Osurua, P.M.B 1200.Ikot Ekpene, Akwa Ibom State, Nigeria 


\begin{abstract}
Metal pollution in freshwater bodies is a long-standing challenge with large expense required to clean-up pollutants such as $\mathrm{Cd}$. There is widespread interest in the potentially low-cost and sustainable use of biological material to perform bioremediation, such as the use of microalgae. Efficient metal bioremediation capacity requires both the ability to tolerate metal stress and metal accumulation. Here, the role of a Chlamydomonas reinhardtii metal tolerance protein (MTP) was examined for enhanced Cd tolerance and uptake. The CrMTP4 gene is a member of the Mn-CDF clade of the cation diffusion facilitator family of metal transporters but is able to provide tolerance and sequestration for $\mathrm{Mn}$ and $\mathrm{Cd}$, but not other metals, when expressed in yeast. Over-expression of CrMTP4 in C. reinhardtii yielded a significant increase in tolerance to $\mathrm{Cd}$ toxicity and increased $\mathrm{Cd}$ accumulation although tolerance to Mn was not increased. In comparison, the metal tolerance of three chlorophyte microalgae strains (Chlorella luteoviridis, Parachlorella hussii, and Parachlorella kessleri) that had previously been adapted to wastewater growth was examined. In comparison to wild type C. reinhardtii, all three natural strains showed significantly increased tolerance to $\mathrm{Cd}, \mathrm{Cu}, \mathrm{Al}$ and $\mathrm{Zn}$, and furthermore their $\mathrm{Cd}$ tolerance and uptake was greater than that of the CrMTP4 over-expression strains. Despite CrMTP4 gene over-expression being a successful strategy to enhance the Cd bioremediation potential of a metal-sensitive microalga, a single gene manipulation cannot compete with naturally adapted strain mechanisms that are likely to be multigenic and due in part to oxidative stress tolerance.
\end{abstract}

Keywords: bioremediation, cadmium uptake, metal tolerance, manganese transport, Chlamydomonas reinhardtii, wastewater 


\section{Introduction}

The potential of using plants and algae for metal bioremediation has led to widespread evaluation of natural species that have innate or adapted ability for metal tolerance and accumulation [1-3]. Furthermore, the genetic engineering of photosynthetic organisms for increased tolerance, uptake, sequestration, transport and chelation of metals has been explored [4]. Macroalgae and unicellular microalgae are particularly attractive for the bioremediation of aquatic environments and wastewaters and a number of natural strains have been identified that are tolerant to a range of metals and show high metal removal abilities [2]. The mechanisms of metal tolerance and accumulation in microalgae appear to be varied, due in part to different responses to different metals, and include metal binding to the cell wall and secreted extracellular polysaccharides, intracellular metal binding peptides and proteins such as phytochelatins, glutathione abundance, oxidative stress tolerance, and metal transporter activity [5-9]. Many of these mechanisms are understood genetically and so there is potential to genetically enhance some of these algal characteristics. However, in contrast to higher plants, so far there have been very few examples of genetic engineering of microalgae to increase metal tolerance and accumulation.

Expression of a chicken class II metallothionein gene in the model green microalga Chlamydomonas reinhardtii led to increased cell growth in the presence of $40 \mu \mathrm{M} \mathrm{Cd}$ and increased Cd binding and removal efficiency relative to wild type, likely due to direct $\mathrm{Cd}$ chelation and sequestration by the metallothionein protein [10]. Chelation of some metals occurs via histidine binding and this has been recently exploited in C. reinhardtii by overexpression of the HISN3 gene, which encodes the enzyme in the fourth step of the histidine biosynthesis pathway. The transgenic microalgae showed an approximately $50 \%$ increase in histidine concentration under $\mathrm{Ni}$ exposure conditions and increased tolerance to Ni stress [11]. Furthermore, the accumulation of metals including $\mathrm{Ni}$, as well as $\mathrm{Zn}, \mathrm{Cu}$ and $\mathrm{Mn}$, was increased in the HISN3 over-expressing C. reinhardtii compared to wild type. In addition to metal binding, metal tolerance can be achieved via anti-oxidant or osmotic protectant activities. Proline accumulation has been linked to the tolerance of a variety of abiotic 
stresses, and increased accumulation of proline in transgenic C. reinhardtii expressing a mothbean $\triangle 1$-pyrroline-5-carboxylate synthetase (P5CS) gene mediated increased $\mathrm{Cd}$ tolerance, possibly due to enhanced anti-oxidant activity and induction of Cd-binding phytochelatin synthesis [12]. All of these examples of microalgal engineering have evaluated improvements to metal chelation and oxidative stress response but as yet there are no examples of metal transporter over-expression in microalgae.

At a cellular level, the manipulation of metal transporter proteins has the potential to enhance metal accumulation into a cell, if metal uptake transporters are over-expressed, or to increase metal tolerance and internal storage, if organelle metal uptake transporters are over-expressed. There are a number of examples in higher plants where this latter strategy has been examined, particularly for tonoplast-localised transporters that perform vacuolar metal sequestration [13-16]. One class of metal transporter involved in metal sequestration and internal metal transport are the Cation Diffusion Facilitators (CDF) that are also called Metal Tolerance Proteins (MTP) in plants and algae [7, 17]. Members of this family transport metal ions like $\mathrm{Zn}^{2+}, \mathrm{Mn}^{2+}, \mathrm{Cd}^{2+}, \mathrm{Co}^{2+}$ and $\mathrm{Fe}^{2+}$, and can be phylogenetically classified within a Zn-CDF, Mn-CDF or Fe/Zn-CDF clade [18]. Many MTPs are vacuolar proteins such as ShMTP8 from Stylosanthes hamata that is responsible for vacuolar $\mathrm{Mn}^{2+}$ sequestration [14], AtMTP3 from Arabidopsis thaliana that can mediate $\mathrm{Zn}^{2+}$ and $\mathrm{Co}^{2+}$ sequestration [19], or CsMTP1 from Cucumis sativus that transports $\mathrm{Zn}^{2+}$ and $\mathrm{Cd}^{2+}[20]$. Others are localised in the secretory pathway, such as the $\mathrm{Mn}^{2+}$ transporting AtMTP11 [21], or at the plasma membrane, such as the $\mathrm{Mn}^{2+}$ and $\mathrm{Cd}^{2+}$ transporting CsMTP9 [22]. In many of these studies, increased expression of an MTP gene often in yeast, led to enhanced cellular metal accumulation and tolerance to high metal concentration, indicating that MTP genes are potential targets for bioremediation studies.

Five MTP genes have been predicted in the C. reinhardtii genome [7] and one of these, CrMTP1 was shown to be transcriptionally induced under $\mathrm{Zn}$ deficiency conditions [23] while CrMTP2 and CrMTP4 are induced by Mn deficiency [24]. However, none of the microalgae MTP genes have yet been directly functionally characterised or evaluated as a 
target for genetic manipulation. Here we describe the cloning and characterisation of CrMTP4 and the evaluation of C. reinhardtii lines over-expressing this gene with regard to $\mathrm{Mn}$ and $\mathrm{Cd}$ tolerance and transport ability. In addition, these transgenic strains were compared with natural strains of chlorophyte microalgae that had been previously obtained from a metal-containing municipal wastewater environment; Chlorella luteoviridis and Parachlorella hussii [25], or had been acclimated under laboratory conditions to tolerate wastewater; as with Parachlorella kessleri [26]. All three strains had been found to tolerate wastewater conditions in part due to increased oxidative stress tolerance, but the specific abilities of these strains to tolerate and accumulate metals has not been previously examined.

\section{Materials and methods}

\subsection{Microalgae strains and growth conditions}

C. reinhardtii wild type strain CC125 was obtained from the Chlamydomonas Resource Center. P. kessleri (CCAP 211/11G) was originally obtained from the UK Culture Collection of Algae and Protozoa (CCAP), Oban, Scotland, UK and was subsequently acclimated for growth in municipal secondary-treated wastewater conditions as described previously [26]. C. Iuteoviridis and $P$. hussii were previously obtained from a municipal wastewater secondary treatment pond as described previously [25]. C. reinhardtii strains over-expressing CrMTP4 were generated as described below. Strains were grown photoheterotrophically in batch culture in Tris-acetate-phosphate (TAP) medium at pH 7 [27] in $200 \mathrm{ml}$ glass flasks on an orbital shaker rotating at $2 \mathrm{~Hz}$ or in $50 \mathrm{ml}$ Nunc flasks, at $25^{\circ} \mathrm{C}$ under cool-white fluorescent lights $\left(150 \mu \mathrm{mol} \mathrm{m}^{-2} \mathrm{~s}^{-1}\right)$ with a $16-\mathrm{h}: 8-\mathrm{h}$ light:dark regime. For metal tolerance and accumulation experiments strains were grown in TAP media supplemented with various concentrations of $\mathrm{Al}_{2}\left(\mathrm{SO}_{4}\right)_{3}, \mathrm{CdCl}_{2}, \mathrm{CuSO}_{4}, \mathrm{MnCl}_{2}, \mathrm{ZnSO}{ }_{4}$ as indicated in the Results section. All cultures were inoculated with the same starting cell density as determined by cell counting to give an initial cell count of $\sim 65 \times 10^{3}$ cells $\mathrm{ml}^{-1}$. 


\subsection{MTP4 cloning and bioinformatic analysis}

C. reinhardtii MTP4 (Cre03.g160550) sequence and gene model information was obtained from Phytozome v.9.1 using v.5.3 of the C. reinhardtii genome annotations. Phylogenetic relationship at the amino acid level was performed using full length sequences, as described previously [28]. The genome ID numbers or accession numbers for the sequences used are: AtMTP1 (At2g46800), AtMTP3 (At3g58810), AtMTP6 (At2g47830), AtMTP7 (At1g51610), AtMTP8 (At3g58060), AtMTP11 (At2g39450), CsMTP1 (EF684941), CsMTP9 (AFJ24702), OsMTP1 (Os05g03780), OsMTP8.1 (Os03g12530), PtrMTP11.1 (EF453693), ShMTP8 (AY181256), ScZRC1 (YMR243C), ScCOT1 (YOR316C), ScMSC2 (YDR205W), ScMMT1 (YMR177W). RNA was isolated from exponential growing C. reinhardtii CC125 cells using Trizol reagent (Life Technologies) and further purified by phenol/chloroform extraction and precipitation with isopropanol. The full length CrMTP4 cDNA (1,617 bp) was amplified by RT-PCR using $1 \mu \mathrm{g}$ of DNase-treated RNA using Superscript III reverse transcriptase (Life Technologies) and an oligo(dT) primer, then KAPA HiFi DNA polymerase (Kapa Biosystems) and gene-specific primers MTP4XbalF (5'-AAA TCT AGA ATG TCG CAA CTA ACG CGC GAA G-3'; Xbal restriction enzyme site underlined) and MTP4SacIR (5'- AAA GAG CTC TCA CAG CAG ATT GAG AGC CTC GCT G-3'; Sacl restriction enzyme site underlined). Genomic DNA was isolated from C. reinhardtii CC125 as described previously [29]. A CrMTP4 genomic DNA fragment spanning the exon and intron regions (3,067 bp) was amplified using KAPA HiFi DNA polymerase and the MTP4XbalF/MTP4SacIR primers. For all PCR amplification conditions, an annealing temperature of $60^{\circ} \mathrm{C}$ and 35 amplification cycles were used. Following amplification, the PCR products were cloned into pGEM-T Easy plasmid (Promega) for propagation and sequencing (GATC Biotech) to confirm sequence fidelity. CrMTP4 cDNA was sub-cloned into the Xbal and Sacl sites of the yeast expression plasmid piUGpd [30] to allow expression under control of the constitutive yeast GAPDH promoter and selection of the URA3 gene. CrMTP4 genomic DNA was sub-cloned into the EcoRI site of the Gateway entry plasmid pENTR1A (Life Technologies) for subsequent recombination using an LR Clonase reaction 
(Life Technologies) into the destination plasmid pH2GW7 [31] to allow expression of CrMTP4 in C. reinhardtii under control of the constitutive cauliflower mosaic virus 35S promoter and selection of the Aph7 gene.

\subsection{Yeast heterologous expression and metal tolerance analysis}

Yeast (S. cerevisiae) strains pmr1 (MATa; his $3 \Delta 1$; leu2 $\Delta 0$; met15 $\Delta 0$; ura3 $\Delta 0$; pmr1::kanMX4) (Euroscarf, Frankfurt, Germany) and the corresponding wild type strain BY4741 (MATa; his3 $\Delta 1$; leu2 $\Delta 0$; met15 $\Delta 0$; ura3 $\Delta 0$ ) (Euroscarf) were each transformed using the lithium acetate-polyethylene glycol method with CrMTP4-piUGpd plasmid or empty piUGpd plasmid and grown at $30^{\circ} \mathrm{C}$ in synthetic defined medium minus uracil (SD -Ura) as described previously [32]. Expression of the CrMTP4 cDNA in yeast was confirmed by RTPCR using the internal CrMTP4 primers MTP4F (5'- ACA TGT GTG TGC GGG AGT CG-3') and MTP4R (5'-CTT GTG CCG GTG CAG GGA CC-3') and RNA extracted from yeast using Trizol reagent, then RT-PCR was performed as described above. PCR products were examined on a $1 \%$ agarose gel stained with SafeView (NBS Biologicals). Metal tolerance assays were performed essentially as described previously [33] on solid SD -Ura medium with or without $3 \mathrm{mM} \mathrm{MnCl} 2$ or $100 \mu \mathrm{M} \mathrm{CdCl}_{2}$ metal salts, or in liquid yeast-peptone-dextrose (YPD) medium with or without $100 \mu \mathrm{M} \mathrm{Al}_{2}\left(\mathrm{SO}_{4}\right)_{3}, 75 \mu \mathrm{M} \mathrm{CdCl}_{2}, 1 \mathrm{mM} \mathrm{CoCl}_{2}, 1 \mathrm{mM} \mathrm{CuSO}_{4}, 5$ $\mathrm{mM} \mathrm{MnCl}_{2}$, or $5 \mathrm{mM} \mathrm{ZnSO}_{4}$ metal salts. Internal $\mathrm{Cd}$ and $\mathrm{Mn}$ content in yeast grown in liquid YPD containing $30 \mu \mathrm{M} \mathrm{CdCl}_{2}$ or $2 \mathrm{mM} \mathrm{MnCl}_{2}$, respectively, was determined by inductively coupled plasma atomic emission spectroscopy (ICP-AES) as described previously [34].

\subsection{C. reinhardtii nuclear genome transformation}

The CrMTP4gDNA-pH2GW7 plasmid or empty pH2GW7 plasmid were transformed into C. reinhardtii CC125 using biolistic bombardment as described previously [29]. Lines were selected on TAP agar medium containing $10 \mu \mathrm{g} \mathrm{ml}^{-1}$ hygromycin B and further selected on fresh selection medium. Three of the lines named MTP4-OE1, MTP4-OE2 and MTP4OE3 were studied further and were maintained in selection medium until gene expression 
analysis and metal tolerance assays were performed. Semi-quantitative expression of CrMTP4 was determined in the MTP4-OE strains and the control (empty pH2GW7) strain at day 3 of growth in TAP medium following cell harvest and snap-freezing in liquid $\mathrm{N}_{2}$ then RNA extraction using Trizol reagent and cDNA synthesis as described above. CrMTP4 cDNA expression was confirmed by RT-PCR using the internal MTP4F/MTP4R primers and was compared relative to expression of the constitutive control transcript $C B L P$ using primers CBLPF (5'-CTT CTC GCC CAT GAC CAC-3') and CBLPR (5'-CCC ACC AGG TTG TTC TTC AG-3'). PCR amplification conditions were as described above except that 25 amplification cycles were used. PCR products were examined on a $1 \%$ agarose gel stained with SafeView.

\subsection{Quantitative gene expression analysis}

C. reinhardtii $\mathrm{CC} 125$ was cultured in TAP medium then $\mathrm{CdCl}_{2}(0.1 \mathrm{mM}$ or $0.2 \mathrm{mM})$ or $\mathrm{MnCl}_{2}(0.5 \mathrm{mM}$ or $1 \mathrm{mM})$ was added to day 3 cells and left to incubate for $8 \mathrm{~h}$ before cells were harvested and frozen in liquid $\mathrm{N}_{2}$. RNA was isolated and cDNA was produced as described above. CrMTP4 gene expression was determined by quantitative real-time PCR (qPCR) using the internal MTP4F/MTP4R primers, a SYBR Green core qPCR kit (Eurogentec) and a StepOnePlus machine (ThermoFisher) using the SYBR Green detection program and normalised to CBLP gene expression. Reactions were run in triplicate and qPCR analysis was performed as described previously [29].

\subsection{Microalgae analysis}

At regular intervals over $8 \mathrm{~d}$, cultures were grown in TAP medium with or without added metals and sampled to determine cell density by cell counting using a Nexcelom Cellometer T4 (Nexcelom Biosciences) or total chlorophyll (chlorophyll a+b) concentration as described previously [26]. Metal content in microalgae after $8 \mathrm{~d}$ growth in metal-containing medium was performed following EDTA washing to remove external (cell wall) bound metals by ICP-AES as described previously [35]. The in vivo production of reactive oxygen species 
(ROS) following 0.4 mM Cd treatment was quantified using the fluorescent stain 2', $7^{\prime}-$ dichlorofluorescein diacetate (DCFH-DA) (Sigma Aldrich) as described previously [25]. Statistical analysis was performed by one-way or two-way ANOVA, as appropriate and Tukey post-hoc test using GraphPad Prism v.6.

\section{Results}

3.1. CrMTP4 is a Mn-CDF that can provide Mn and Cd tolerance and uptake in yeast Previous analysis of the $C$. reinhardtii genome has identified five MTP genes that are members of the CDF family [7]. CrMTP4 is suggested to be a $\mathrm{Mn}^{2+}$-transporting CDF member but the transport specificity of this protein is unknown. Phylogenetic analysis of amino acid sequence indicates that CrMTP4 does indeed fall within the Mn-CDF clade alongside other known $\mathrm{Mn}^{2+}$-transporting MTPs including ShMTP8, AtMTP11, OsMTP8, and the $\mathrm{Mn}^{2+} / \mathrm{Cd}^{2+}$ transporting CsMTP9, although it is distinctly clustered from the higher plant Mn-CDF proteins (Fig. 1a). To examine whether CrMTP4 transcriptionally responds to $\mathrm{Mn}$ or Cd excess in vivo, day 3 C. reinhardtii CC125 cells were examined following $8 \mathrm{~h}$ treatment with $\mathrm{Mn}$ addition (up to $1 \mathrm{mM}$ ) or $\mathrm{Cd}$ addition (up to $0.2 \mathrm{mM}$ ), however, there was no significant difference in CrMTP4 transcript abundance between treatments (Fig. 1b) demonstrating that CrMTP4 expression is not transcriptionally regulated by $\mathrm{Cd}$ or excess $\mathrm{Mn}$ status. The full length CrMTP4 cDNA was obtained by RT-PCR and metal tolerance function was confirmed by yeast heterologous expression. When expressed in the Mn sensitive yeast mutant strain pmr1, CrMTP4 expression was able to suppress the Mn sensitivity and provide strong Mn tolerance (Fig. 1c). Following expressing in a wild type yeast strain, CrMTP4 was able to increase tolerance to $\mathrm{Cd}$ (Fig. 1c and d), however, there was no change in the ability of CrMTP4-expressing yeast to grow in excess concentrations of other metals including Zn, Co, Cu or Al (Fig. 1d). The Mn and Cd tolerance provided by CrMTP4 expression in yeast appeared to be due to internal sequestration as total cellular concentration of $\mathrm{Mn}$ and $\mathrm{Cd}$ was significantly increased in the CrMTP4 yeast compared to the control, by 2.48 -fold for Mn and 2.29-fold for Cd (Fig. 1e). 


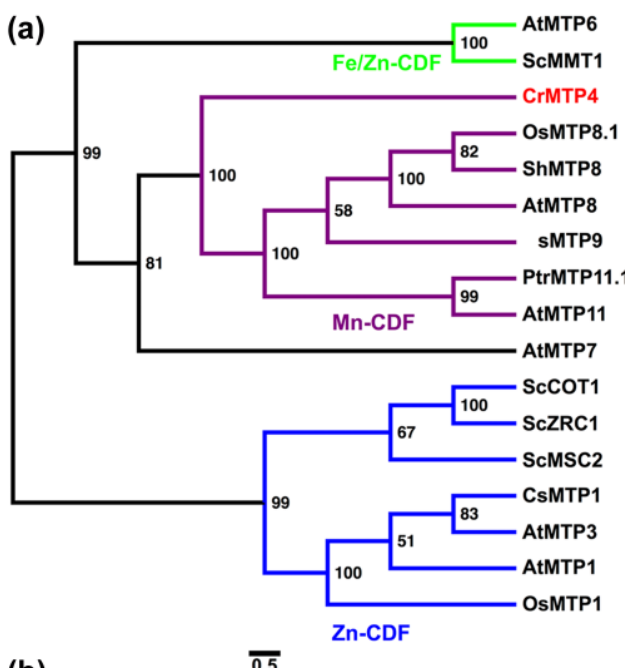

(b)

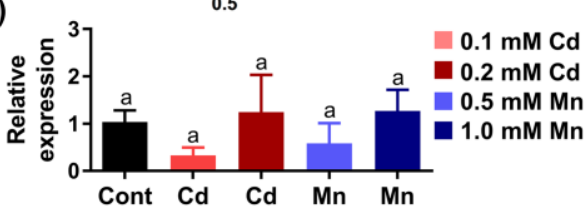

(c) -Ura
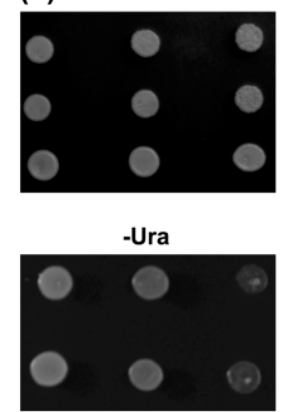

(d)

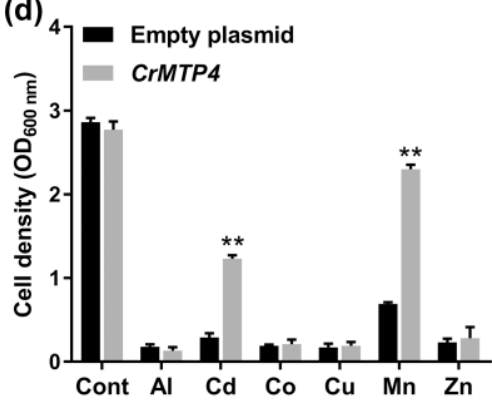

$+3 \mathrm{mM} \mathrm{Mn}$

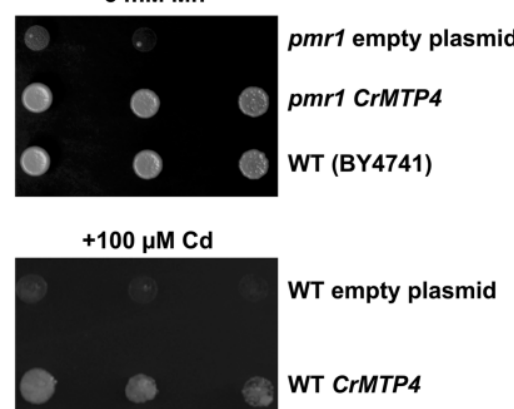

(e)

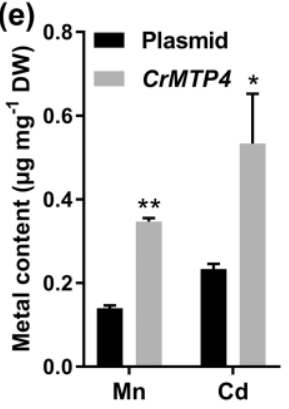

Fig. 1. Cloning of CrMTP4 and characterization by yeast heterologous expression. (a) Phylogenetic comparison of CrMTP4 with selected Mn-CDF, Zn-CDF and Fe/Zn-CDF proteins from Arabidopsis thaliana (At), Cucumis sativus (Cs), Oryza sativa (Os), Populus trichocarpa x P. deltoides (Ptr), Stylosanthes hamata (Sh) and Saccharomyces cerevisiae (Sc). A maximum likelihood tree was derived from full length amino acid sequence alignment. Bootstrap values from 100 replications are indicated and the branch length scale bar indicates the evolutionary distance of 0.5 amino acid substitution per site. (b) Gene expression of CrMTP4 in wild type cells in response to $\mathrm{Cd}$ and Mn treatment. Expression of CrMTP4 as determined by real-time $\mathrm{PCR}$ is shown relative to $C B L P$ expression. Data points are means $( \pm \mathrm{SE})$ calculated from 3 independent biological replicates. Bars sharing the same lowercase letter indicate no significant difference between treatments $(P>0.05)$. (c) Suppression of Mn sensitivity of the pmr1 yeast mutant, and Cd sensitivity of wild type (BY4741) yeast, both expressing CrMTP4 in comparison with empty plasmid-containing strains. Liquid cultures of strains were serially diluted then spotted onto SD -Ura medium with or without added $\mathrm{Mn}$ or $\mathrm{Cd}$. Yeast growth is shown after $3 \mathrm{~d}$. A representative experiment is shown. (d) BY4741 strains transformed with empty plasmid or CrMTP4 normalized to an identical starting cell density then grown in liquid YPD medium with or without added metals $(0.1 \mathrm{mM} \mathrm{Al}, 75 \mu \mathrm{M} \mathrm{Cd}, 1 \mathrm{mM} \mathrm{Co}, 1 \mathrm{mM} \mathrm{Cu}, 5 \mathrm{mM} \mathrm{Mn}, 5 \mathrm{mM} \mathrm{Zn})$ and grown for $24 \mathrm{~h}$. Cell density was determined by optical density measurement at $600 \mathrm{~nm}$. (e) Mn and Cd uptake in EDTA-washed BY4741 yeast transformed with empty plasmid or CrMTP4 following growth in $2 \mathrm{mM} \mathrm{Mn}$ or $30 \mu \mathrm{M}$ Cd YPD media. Data points are means ( \pm SE) of 3 independent biological replicates. Bars indicated by asterisks show significant difference between empty plasmid and CrMTP4 strain within each metal treatment $\left({ }^{*}, P<0.05 ;{ }^{* *}, P<0.01\right)$.

\subsection{Over-expression of CrMTP4 in C. reinhardtii enhances Cd tolerance}

\section{A genomic construct of CrMTP4 containing all predicted exons and introns was} transformed into the nuclear genome of wild type C. reinhardtii CC125 and three lines 
showing strong hygromycin tolerance and significantly higher expression of CrMTP4 relative to wild type level of CrMTP4 expression (Fig. 2a) were chosen for further analysis. There was no significant difference in cell density or chlorophyll concentration in any of the CrMTP4 over-expression lines compared to wild type under standard growth conditions (Fig. $2 \mathrm{~b}$ and $\mathrm{c})$. Furthermore, while $\mathrm{Mn}$ addition to the medium up to $2 \mathrm{mM}$ inhibited cell growth (Fig. 2b) and chlorophyll concentration (Fig. 2c) there was no increase in tolerance to Mn by any of the CrMTP4 lines. However, there was evidence of a subtle but significant increase in Mn accumulation in two of the three CrMTP4 lines (CrMTP4-OE2 and CrMTP4-OE3) compared to wild type, by 1.9 - and 2.3-fold, respectively (Fig. 2d).

(a)

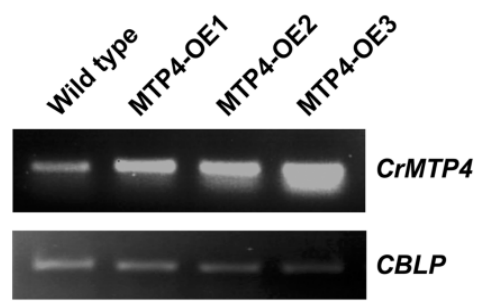

(b)

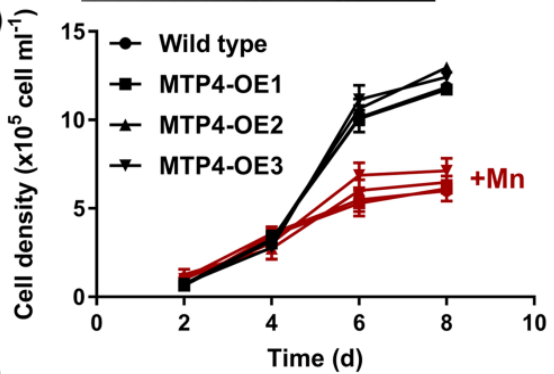

(c)
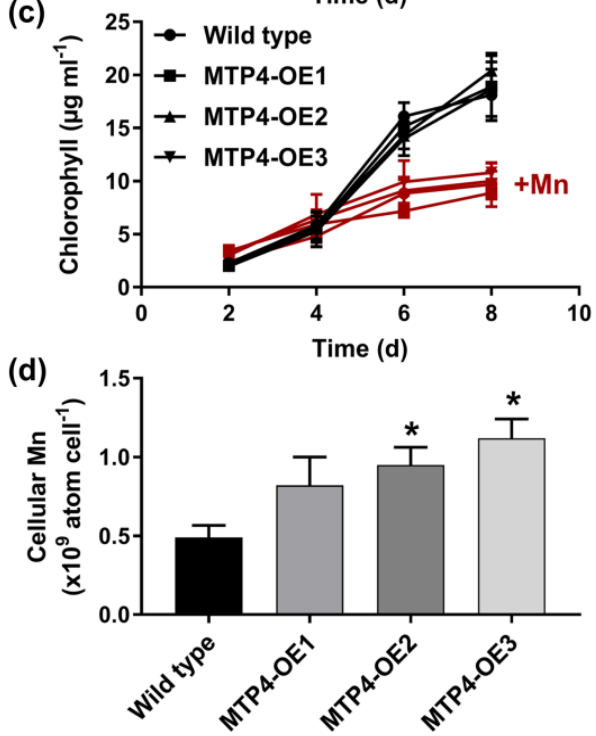

Fig. 2. Generation of transgenic CrMTP4 over-expression lines with enhanced Mn accumulation. (a) RT-PCR detection of CrMTP4 mRNA transcript abundance in wild type cells in comparison to three independent CrMTP4 overexpression (MTP4OE) lines. RT-PCR was performed using CBLP as a normalization control transcript. (b and c) Cell density as determined by 
cell count measurement (b) and total chlorophyll yield (c) in wild type and MTP-OE lines over time in TAP medium with (red symbols) or without (black symbols) $2 \mathrm{mM} \mathrm{Mn}$ addition. (d) Cellular Mn content in EDTA-washed wild type and MTP-OE cells following growth in $2 \mathrm{mM} \mathrm{Mn}$ medium after $8 \mathrm{~d}$. Data points are means $( \pm \mathrm{SE})$ of 3 independent biological replicates. Bars indicated by asterisks show significant difference between wild type and MTP-OE line $\left({ }^{*}, P<0.05\right)$.

In contrast, all three CrMTP4 lines exhibited significant tolerance to Cd addition (Fig. 3a). While the wild type cells displayed a marked loss in cell density and chlorosis in all Cd treatments, growth and total chlorophyll yield of the CrMTP4 lines was significantly enhanced but there was no significant difference between the individual CrMTP4 lines (Fig. $3 \mathrm{~b}$ and c). In $0.5 \mathrm{mM} \mathrm{Cd}$ conditions, the wild type strain was unable to grow while CrMTP4 over-expression strains grew strongly (Fig. 3b), although growth was still significantly inhibited compared to treatments without Cd addition (Fig. 3b). To examine whether the increased stress of the strains in response to Cd addition was associated with oxidative stress, the reporter DCFH-DA for intracellular ROS production was used. C. reinhardtii strains were grown in TAP medium with $0.4 \mathrm{mM} \mathrm{Cd}$ addition and ROS production was measured in day 4 cells relative to cells with no added Cd. There was a $152 \pm 26 \%$ increase in ROS production in wild type and a $138 \pm 32 \%$ increase in the CrMTP4 over-expression line but there was no significant difference between the strains. Growth of the CrMTP4 overexpression lines was compared on other metal treatment conditions including $\mathrm{Zn}, \mathrm{Cu}$ and $\mathrm{Al}$ toxicity but there was no difference in growth compared to wild type, indicating that the CrMTP4 over-expression metal tolerance trait is specific for $\mathrm{Cd}$. 
(a)

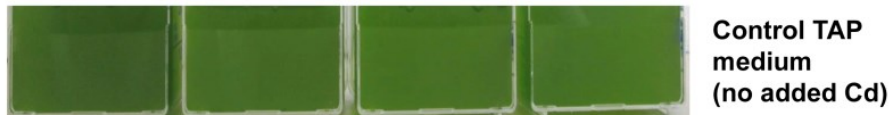

$\int()_{1}$

(no added $\mathrm{Cd}$ )

$+0.4 \mathrm{mM} \mathrm{Cd}$

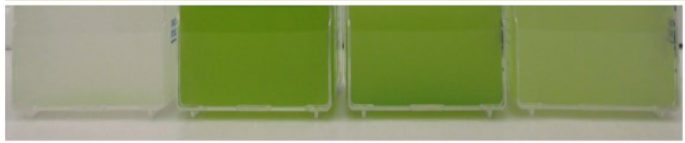

$+0.5 \mathrm{mM} \mathrm{Cd}$

Wild type MTP4-OE1 MTP4-OE2 MTP4-OE3

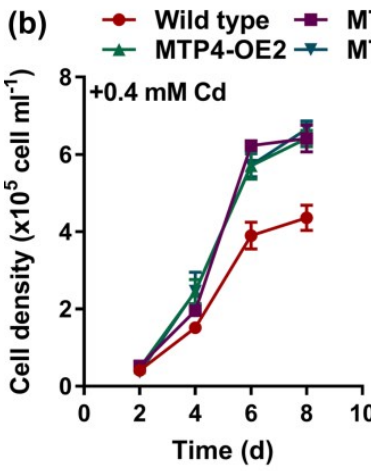

MTP4-OE1

\section{P4-OE3}
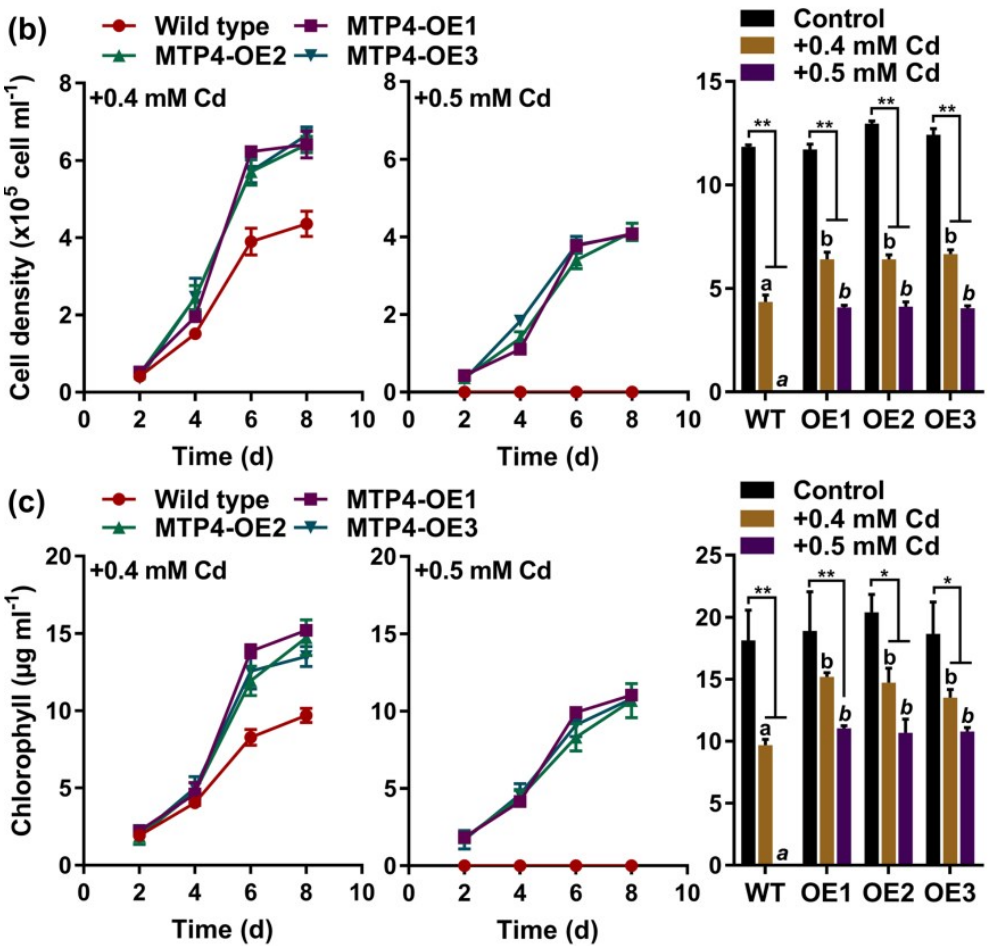

\section{TP4-OE3}

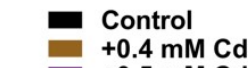

$207+0.5 \mathrm{mMCd}$

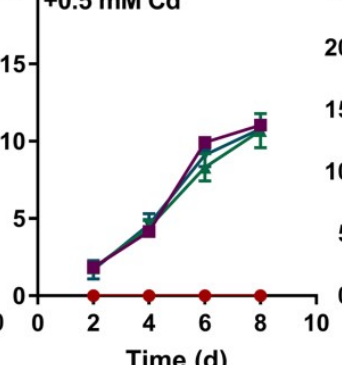

$+0.5 \mathrm{mMCd}$

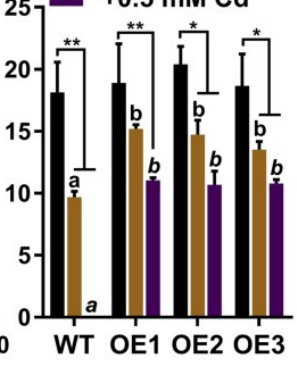

Fig. 3. Cd tolerance of CrMTP4 over-expression lines. (a) Culture phenotypes of wild type C. reinhardtii and CrMTP4 overexpression (MTP4-OE) lines after $7 \mathrm{~d}$ growth in medium with or without Cd. A representative experiment is shown. (b and c) Cell density as determined by cell count measurement (b) and total chlorophyll yield (c) in wild type and MTP-OE lines over time in TAP medium with 0.4 or $0.5 \mathrm{mM} \mathrm{Cd}$ addition. Cell density and chlorophyll parameters are shown in comparison to control treatment without $\mathrm{Cd}$ addition after $8 \mathrm{~d}$ growth (right-hand panels). Data points are means $( \pm \mathrm{SE})$ of 3 independent biological replicates. Bars indicated by different lower case letters show significant difference $(P<0.05)$ within Cd treatments and between wild type (WT) and MTP-OE lines. Bars indicated by asterisks show significant difference between control and Cd treatments $\left({ }^{*}, P<0.05 ;{ }^{* *}, P<0.01\right)$.

\subsection{Natural wastewater adapted microalgae strains provide more substantial Cd tolerance and accumulation than CrMTP4 C. reinhardtii}

Microalgae that are found in polluted environments such as in wastewater are adapted over many generations to the pollution conditions and therefore might be expected 
to perform better with regard to metal tolerance and bioremediation than non-adapted strains. However, it is unclear how a transgenic line such the CrMTP4 overexpression strain would compare against an adapted strain. Three microalgae strains of species $C$. luteoviridis, $P$. hussii and $P$. kessleri, were previously isolated from, or artificially acclimated to, municipal wastewater conditions $[25,26]$. This secondary-treated wastewater was the outflow from an activated sludge treatment at a facility receiving mainly domestic wastewater and pre-treated effluent from a nearby oil refinery. The primary sedimentation and activated sludge treatment had reduced the concentration of suspended solids, biochemical oxygen demand/chemical oxygen demand, and ammonium and phosphate, but at this stage the wastewater conditions still included exposure to high concentrations of ammonium (22.7 $\left.30.1 \mathrm{mg} \mathrm{L}^{-1}\right)$ and phosphate $\left(1.4-1.7 \mathrm{mg} \mathrm{L}^{-1}\right)$, bacterial contamination, and high oxygen concentration. Trace metals were present in the wastewater although their concentrations were fairly low (0.9 $\mu \mathrm{M}$ Cd; $2.7 \mu \mathrm{M} \mathrm{Cu} ; 125.2 \mu \mathrm{M}$ Fe; $16.7 \mu \mathrm{M} \mathrm{Mn;} 18.8 \mu \mathrm{M} \mathrm{Zn})$. Nevertheless, the three strains showed substantial tolerance to multiple metals compared to wild type and non-adapted C. reinhardtii. The tolerance to $150 \mu \mathrm{M} \mathrm{Cd}$ addition, as determined by chlorophyll content, for all three natural strains, especially $P$. hussii, was significantly higher compared to wild type $C$. reinhardtii but also significantly higher compared to the CrMTP4 over-expression strains (Fig. 4a). However, it was seen that even under non-stressed (no added metal) conditions, the three wastewater-adapted strains grew better than the C. reinhardtii strains on the basis of total chlorophyll content. Normalized to non-stressed treatments, all three of the wastewater adapted strains still showed significantly greater tolerance than the wild type $C$. reinhardtii, while $P$. hussii showed significantly greater tolerance compared to the CrMTP4 over-expression strains (Fig. 4a inset). CrMTP4 over-expression lines were able to accumulate significantly more Cd than wild type (2.81- to 3.06-fold higher) but Cd accumulation was also significantly higher for the wastewateradapted strains compared to both wild type and CrMTP4 over-expressing C. reinhardtii; C. luteoviridis, P. hussii and P. kessleri displayed 9.7-fold, 8.6-fold, and 7.3-fold higher accumulation compared to wild type C. reinhardtii, respectively (Fig. 4b). For this analysis, 
Cd concentration was determined in EDTA-washed cells in order to remove cell wall-bound metal and therefore determine internalised metal uptake.
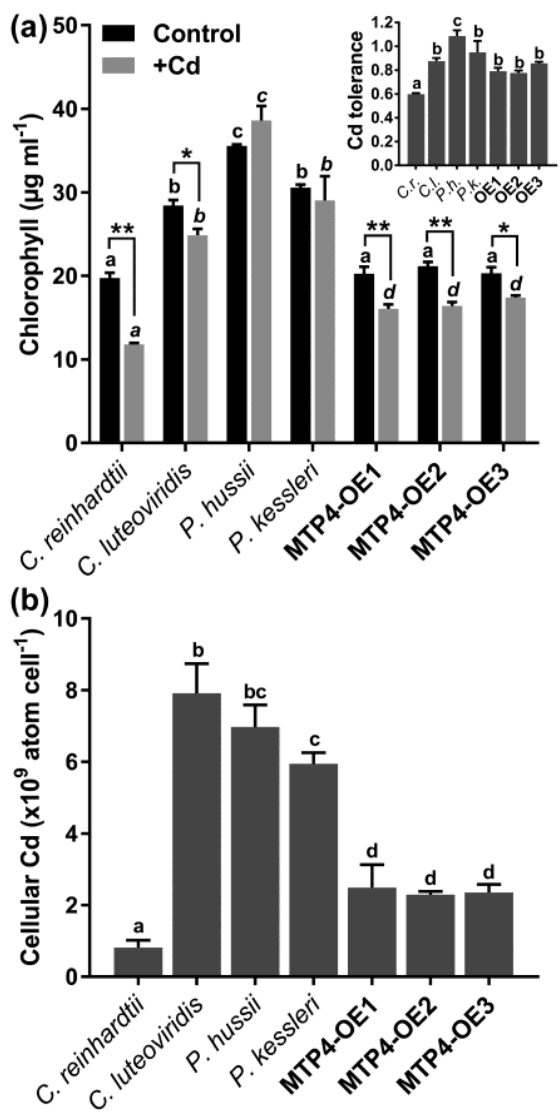

Fig. 4. Cd tolerance and accumulation of CrMTP4 over-expression lines in comparison to natural wastewater adapted microalgae strains. (a) Total chlorophyll yield of strains after $8 \mathrm{~d}$ of growth in TAP medium with or without (control) $150 \mu \mathrm{M} \mathrm{Cd}$ addition. Inset: normalized chlorophyll yield of strains following Cd treatment relative to treatment without Cd. (b) Cellular Cd content in EDTA-washed cells following growth in $150 \mu \mathrm{M}$ Cd medium after $8 \mathrm{~d}$. Data points are means $( \pm S E)$ of 3 independent biological replicates. Bars indicated by different lower case letters show significant difference $(P<0.05)$ within treatments, and pairs of bars indicated by asterisks show significant difference between control and $\mathrm{Cd}$ treatment $\left({ }^{*}, P<0.05 ;{ }^{* *}, P<0.01\right)$.

While the CrMTP4 over-expression strains were specifically enhanced for Cd tolerance and accumulation, the three wastewater-adapted strains also displayed increased tolerance to other metals including $\mathrm{Al}, \mathrm{Cu}$ and $\mathrm{Zn}$ compared to wild type $\mathrm{C}$. reinhardtii, in terms of cell density (Fig. 5a) and chlorophyll content (Fig. 5b), including when normalized to values without metal addition (Fig. 5a inset and 5b inset). For all metal conditions, on the basis of cell density, $P$. hussii was the most tolerant microalga, while $P$. kessleri showed high metal tolerance on the basis of chlorophyll content. The internalised accumulation of $\mathrm{Al}, \mathrm{Cu}$ 
and $\mathrm{Zn}$ was also significantly higher for all three strains, with the exception of $\mathrm{Zn}$

accumulation by $C$. Iuteoviridis, compared to $C$. reinhardtii, and with $P$. kessleri having the highest accumulation of all three metals (Fig. 5c).
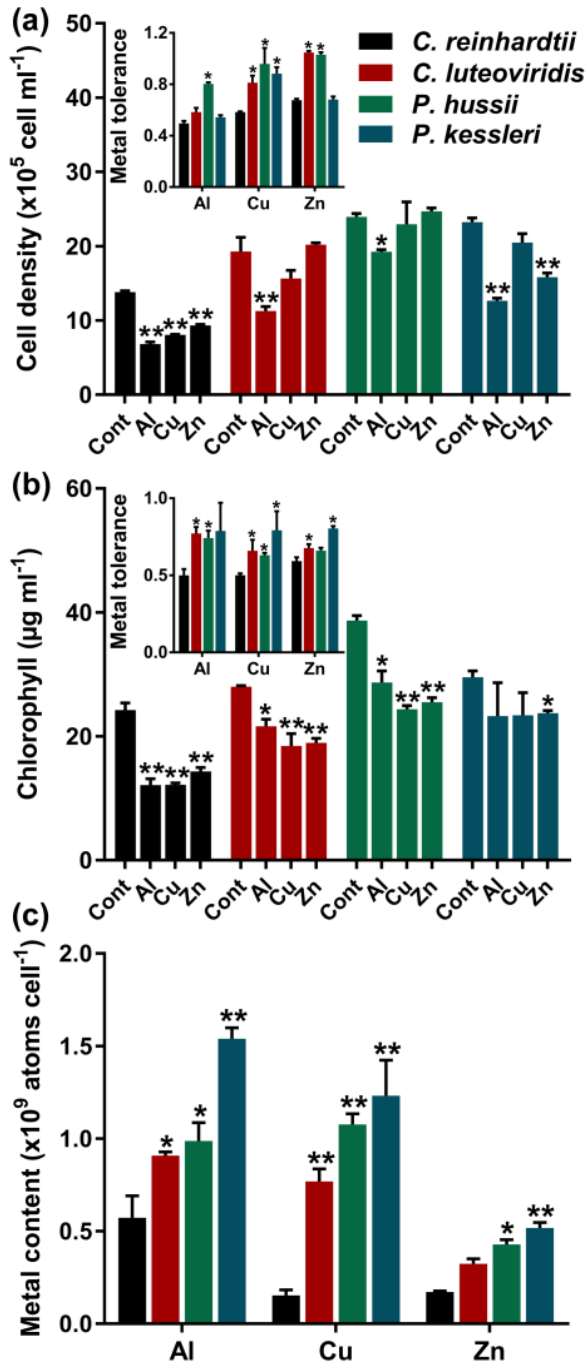

Fig. 5. Metal tolerance and accumulation characteristics of natural wastewater adapted microalgae strains. (a and b) Cell density (a) and total chlorophyll yield (b) of natural strains treated without metal addition (Cont.) or with $100 \mu \mathrm{M} \mathrm{Al,} 100 \mu \mathrm{M}$ Cu, and $225 \mu \mathrm{M} Z \mathrm{n}$ in TAP medium for $8 \mathrm{~d}$. Inset graphs: normalized cell density (a) and chlorophyll yield (b) of strains following metal treatment relative to treatment without metals. (c) Cellular metal content in EDTA-washed cells following growth in $50 \mu \mathrm{M}$ Al, $50 \mu \mathrm{M} \mathrm{Cu}$ and $150 \mu \mathrm{M}$ Zn medium after $8 \mathrm{~d}$. Data points are means $( \pm \mathrm{SE})$ of 3 independent biological replicates. Bars indicated by asterisks show significant difference between metal treatment and control treatment, or between $C$. reinhardtii and the wastewater adapted strain $\left({ }^{*}, P<0.05 ;{ }^{* *}, P<0.01\right)$. 


\section{Discussion}

Mn-CDF genes have been identified and characterized from various higher plant species but have not as yet been examined in much detail from microalgae. Furthermore, there have been very few studies examining the bioremediation potential of MTP gene targets, and none using microalgae. Here we have functionally determined the substrate specificity of a Mn-CDF, CrMTP4 from C. reinhardtii and demonstrated the ability to enhance Cd tolerance and accumulation in this species of microalgae through over-expression of this $\mathrm{Mn}-\mathrm{CDF}$.

Phylogenetic analysis showed that CrMTP4 groups with other known $\mathrm{Mn}^{2+}$ transporting MTP proteins, while previous experiments showing induction of CrMTP4 mRNA transcript under Mn deficiency conditions had implicated this putative MTP as a $\mathrm{Mn}^{2+}$ transporter [24]. Yeast heterologous expression clearly confirmed the ability of CrMTP4 to increase the cellular accumulation of $\mathrm{Mn}^{2+}$ as well as to enhance tolerance of $\mathrm{Mn}$ in yeast, possibly due to vacuolar sequestration although this could not be confirmed. Furthermore, the yeast assay suggested that CrMTP4 also displayed $\mathrm{Cd}^{2+}$ transport and tolerance activity but it could not provide yeast tolerance to other metals tested including $\mathrm{Zn}^{2+} . \mathrm{Cd}^{2+}$ is a nonessential metal ion for photosynthetic organisms but is readily taken up and transported throughout cells often in competition with essential metal ions such as $\mathrm{Zn}^{2+}$ [36], and indeed some $\mathrm{Zn}-\mathrm{CDF}$ proteins such as OsMTP1 have been implicated in the transport of both $\mathrm{Zn}^{2+}$ and $\mathrm{Cd}^{2+}[37]$. Many of the previously studied Mn-CDF proteins such as ShMTP8, AtMTP8, and AtMTP11 appear to be specific for $\mathrm{Mn}^{2+}[14,21,38]$, but it is possible for $\mathrm{Mn}^{2+}$ transporters to also be able to transport $\mathrm{Cd}^{2+}$. For example, the vacuolar $\mathrm{Mn}^{2+}$ and $\mathrm{Ca}^{2+}$ transporter AtCAX2 is also able to transport $\mathrm{Cd}^{2+}[16]$, while the Mn-CDF transporter CsMTP9 was also shown to be able to transport $\mathrm{Cd}^{2+}[22]$. As a $\mathrm{Mn}^{2+}$ and $\mathrm{Cd}^{2+}$ transporter that was able to increase metal accumulation when expressed in yeast, CrMTP4 was therefore a suitable target to examine bioremediation potential by over-expression in $C$. reinhardtii. 
A genomic construct of CrMTP4 led to increased CrMTP4 mRNA transcript abundance in C. reinhardtii, which had no obvious negative effects to the cells under nonstressed conditions. However, unlike in yeast, increased expression of CrMTP4 was unable to provide tolerance to excess $\mathrm{Mn}$, despite the transgenic cells exhibiting a slight increase in Mn accumulation. Although CrMTP4 was able to promote Mn tolerance in a heterologous system, the $\mathrm{Mn}^{2+}$ transport and homeostasis characteristics may be different in vivo. Allen et al. (2007) [24] demonstrated that out of all five C. reinhardtii MTP genes, CrMTP4 showed the highest induction in response to Mn deficiency, perhaps indicating that this gene encodes a high affinity $\mathrm{Mn}^{2+}$ transporter responsible for $\mathrm{Mn}^{2+}$ delivery under situations of low Mn availability and therefore is poorly suited to conditions of high Mn stress. In fact we found that CrMTP4 was not transcriptionally induced by excess concentrations of $\mathrm{Mn}$, or indeed Cd. Alternatively, this may suggest that CrMTP4 is not directly regulated by toxic concentrations of metals or possibly that regulation is post-transcriptional. While some higher plant MTP genes such as CsMTP9 are transcriptionally induced by metal excess [22], others such as AtMTP11 and OsMTP8.1 are not significantly induced by high concentrations of $\mathrm{Mn}[21,39]$, although OsMTP8.1 protein abundance is moderately enhanced by high Mn supply. It is interesting to note that like for CrMTP4, the yeast Zn-CDF gene ScZRC1 is also transcriptionally induced by metal limitation but shows low expression in response to high metal conditions, despite being an important component in $\mathrm{Zn}$ tolerance [40]. It was suggested that this is a proactive protective mechanism against a sudden switch from metal limited to excess conditions. Finally it is worth noting that the sub-cellular localization of CrMTP4 was not determined and the organelle or vesicle on which CrMTP4 resides may provide insufficient internal $\mathrm{Mn}$ storage to tolerate sequestration of high concentrations of $\mathrm{Mn}^{2+}$. Unlike higher plants and yeast, C. reinhardtii does not possess a large central vacuole but many smaller acidic vacuoles or acidocalcisomes [41, 42]. This potentially argues that internal metal sequestration as a bioremediation mechanism may be less useful for microalgae. 
In contrast to the Mn stress response, the MTP4-OE lines all showed increased tolerance to increasing concentrations of $\mathrm{Cd}$, up to $0.5 \mathrm{mM} \mathrm{Cd}$, coupled with increased cellular content of Cd compared to wild type, suggesting internal sequestration of Cd rather than $\mathrm{Cd}$ efflux. In comparison to other previously generated transgenic $\mathrm{Cd}$ tolerant $\mathrm{C}$. reinhardtii strains, the MTP4-OE lines displayed greater tolerance to Cd than the prolineaccumulating P5CS lines that only showed a 1.5-fold increase in growth compared to wild type grown in $100 \mu \mathrm{M}$ Cd [12]. The MTP4-OE lines also had greater Cd tolerance compared to C. reinhardtii expressing a chicken metallothionein [10]. To date, this is the only example of an MTP gene being genetically engineered in an algal species to enhance potential metal bioremediation characteristics, and there are just two recent examples to compare of an MTP being over-expressed in higher plants. OsMTP1 was ectopically expressed in tobacco to give increased tolerance to $100 \mu \mathrm{M} \mathrm{Cd}$, by supressing inhibition of growth, lipid peroxidation and cell death compared to wild type tobacco [43]. Furthermore, OsMTP1 expression gave an approximately 2 -fold increase in whole plant Cd accumulation compared to wild type, with the tolerance and accumulation trait likely due to increased vacuolar sequestration. Likewise, ectopic expression of CsMTP9 in A. thaliana increased plant tolerance to $\mathrm{Cd}$ and led to enhanced shoot $\mathrm{Cd}$ accumulation relative to the roots [22]. Unlike for CrMTP4, CsMTP4 over-expression also led to increased Mn tolerance, however, it is important to note that CsMTP4 localized to the plasma membrane and provides metal tolerance by cellular efflux.

Despite the increased Cd tolerance and accumulation provided by CrMTP4 overexpression, the natural wastewater adapted strains all showed substantially greater tolerance and accumulation of $\mathrm{Cd}$, as well as other metals including $\mathrm{Al}, \mathrm{Cu}$ and $\mathrm{Zn}$. Previous analysis of these natural strains demonstrated that they are able to tolerate the toxic effects of the wastewater environment because of high oxidative stress tolerance activities, which include increased ascorbate peroxidase activity and carotenoid accumulation compared to the non-adapted strains [25, 26]. Prolonged exposure to metals such as Cd induces cellular toxicity in part due to oxidative stress $[44,45]$. Indeed it was also demonstrated here that 
ROS accumulation was increased in C. reinhardtii strains in response to the Cd treatment, however, there was no significant difference in ROS production following CrMTP4 overexpression, suggesting that the increased Cd tolerance provided by enhanced CrMTP4mediated Cd transport activity was not due to reduced ROS accumulation. Although it cannot be ruled out that the wastewater-adapted $P$. hussii, $P$. kessleri and C. luteoviridis strains have higher abundance and activity of other metal tolerance mechanisms, such as metal transporters, compared to $C$. reinhardtii, these results suggest that oxidative stress tolerance activity is a better target in microalgae than enhanced metal sequestration alone. This also suggests that future genetic engineering strategies for microalgae metal bioremediation should additionally focus on manipulating oxidative stress tolerance. Moreover with potential concerns about the risks of genetically modified microalgae escape into the environment as well as regulatory restrictions [46], there is arguably currently greater attraction for natural strains rather than transgenic strains.

\section{Acknowledgements}

This work was supported in part by The Leverhulme Trust (grant number F/00 120/BG to J.K.P.) and a BBSRC Activating Impact award. A.I. thanks the Government of Nigeria TETFUND for PhD studentship funding. We thank Paul Lythgoe (School of Earth and Environmental Sciences, University of Manchester) for ICP-AES analysis and thank Olumayowa Osundeko for providing wastewater metal concentration values.

\section{References}

[1] U. Krämer, Metal hyperaccumulation in plants, Annu. Rev. Plant Biol., 61 (2010) 517-534.

[2] K. Suresh Kumar, H.-U. Dahms, E.-J. Won, J.-S. Lee, K.-H. Shin, Microalgae - A promising tool for heavy metal remediation, Ecotoxicol. Environ. Saf., 113 (2015) 329-352. [3] S.K. Mehta, J.P. Gaur, Use of algae for removing heavy metal ions from wastewater: progress and prospects, Crit. Rev. Biotechnol., 25 (2005) 113-152. 
[4] D.M. Antosiewicz, A. Barabasz, O. Siemianowski, Phenotypic and molecular consequences of overexpression of metal-homeostasis genes, Front. Plant Sci., 5 (2014) 80. [5] G. Howe, S. Merchant, Heavy metal-activated synthesis of peptides in Chlamydomonas reinhardtii, Plant Physiol., 98 (1992) 127-136.

[6] M. Lavoie, S. Le Faucheur, C. Fortin, P.G.C. Campbell, Cadmium detoxification strategies in two phytoplankton species: Metal binding by newly synthesized thiolated peptides and metal sequestration in granules, Aquat. Toxicol., 92 (2009) 65-75. [7] C.E. Blaby-Haas, S.S. Merchant, The ins and outs of algal metal transport, Biochim. Biophys. Acta, 1823 (2012) 1531-1552.

[8] J.M. Collard, R.F. Matagne, $\mathrm{Cd}^{2+}$ resistance in wild-type and mutant strains of Chlamydomonas reinhardtii, Environ. Exp. Bot., 34 (1994) 235-244.

[9] M. Hanikenne, P. Motte, M.C.S. Wu, T. Wang, R. Loppes, R.F. Matagne, A mitochondrial half-size $A B C$ transporter is involved in cadmium tolerance in Chlamydomonas reinhardtii, Plant Cell Environ., 28 (2005) 863-873.

[10] X.-H. Cai, C. Brown, J. Adhiya, S.J. Traina, R.T. Sayre, Growth and heavy metal binding properties of transgenic Chlamydomonas expressing a foreign metallothionein gene, Int. J. Phytoremediat., 1 (1999) 53-65.

[11] Q. Zheng, Z.Z. Cheng, Z.M. Yang, HISN3 mediates adaptive response of Chlamydomonas reinhardtii to excess nickel, Plant Cell Physiol., 54 (2013) 1951-1962. [12] S. Siripornadulsil, S. Traina, D.P.S. Verma, R.T. Sayre, Molecular mechanisms of proline-mediated tolerance to toxic heavy metals in transgenic microalgae, Plant Cell, 14 (2002) 2837-2847.

[13] Q. Wu, T. Shigaki, K.A. Williams, J.S. Han, C.K. Kim, K.D. Hirschi, S. Park, Expression of an Arabidopsis $\mathrm{Ca}^{2+} / \mathrm{H}^{+}$antiporter $\mathrm{CAX} 1$ variant in petunia enhances cadmium tolerance and accumulation, J. Plant Physiol., 168 (2011) 167-173.

[14] E. Delhaize, T. Kataoka, D.M. Hebb, R.G. White, P.R. Ryan, Genes encoding proteins of the cation diffusion facilitator family that confer manganese tolerance, Plant Cell, 15 (2003) 1131-1142. 
[15] M. Morel, J. Crouzet, A. Gravot, P. Auroy, N. Leonhardt, A. Vavasseur, P. Richaud, AtHMA3, a $\mathrm{P}_{1 \mathrm{~B}}-\mathrm{ATPase}$ allowing $\mathrm{Cd} / \mathrm{Zn} / \mathrm{Co} / \mathrm{Pb}$ vacuolar storage in Arabidopsis, Plant Physiol., 149 (2009) 894-904.

[16] K.D. Hirschi, V.D. Korenkov, N.L. Wilganowski, G.J. Wagner, Expression of Arabidopsis CAX2 in tobacco. Altered metal accumulation and increased manganese tolerance, Plant Physiol., 124 (2000) 125-133.

[17] F.K. Ricachenevsky, P.K. Menguer, R.A. Sperotto, L.E. Williams, J.P. Fett, Roles of plant Metal Tolerance Proteins (MTP) in metal storage and potential use in biofortification strategies, Front. Plant Sci., 4 (2013) 144.

[18] B. Montanini, D. Blaudez, S. Jeandroz, D. Sanders, M. Chalot, Phylogenetic and functional analysis of the Cation Diffusion Facilitator (CDF) family: improved signature and prediction of substrate specificity, BMC Genomics, 8 (2007).

[19] S. Arrivault, T. Senger, U. Kramer, The Arabidopsis metal tolerance protein AtMTP3 maintains metal homeostasis by mediating $\mathrm{Zn}$ exclusion from the shoot under Fe deficiency and Zn oversupply, Plant J., 46 (2006) 861-879.

[20] M. Migocka, A. Kosieradzka, A. Papierniak, E. Maciaszczyk-Dziubinska, E. Posyniak, A. Garbiec, S. Filleur, Two metal-tolerance proteins, MTP1 and MTP4, are involved in Zn homeostasis and Cd sequestration in cucumber cells, J. Exp. Bot., 66 (2015) 1001-1015. [21] E. Delhaize, B.D. Gruber, J.K. Pittman, R.G. White, H. Leung, Y.S. Miao, L.W. Jiang, P.R. Ryan, A.E. Richardson, A role for the AtMTP11 gene of Arabidopsis in manganese transport and tolerance, Plant J., 51 (2007) 198-210.

[22] M. Migocka, A. Papierniak, A. Kosieradzka, E. Posyniak, E. Maciaszczyk-Dziubinska, R. Biskup, A. Garbiec, T. Marchewka, Cucumber metal tolerance protein CsMTP9 is a plasma membrane $\mathrm{H}^{+}$-coupled antiporter involved in the $\mathrm{Mn}^{2+}$ and $\mathrm{Cd}^{2+}$ efflux from root cells, Plant J., 84 (2015) 1045-1058.

[23] D. Malasarn, J. Kropat, S.I. Hsieh, G. Finazzi, D. Casero, J.A. Loo, M. Pellegrini, F.-A. Wollman, S.S. Merchant, Zinc deficiency impacts $\mathrm{CO}_{2}$ assimilation and disrupts copper homeostasis in Chlamydomonas reinhardtii, J. Biol. Chem., 288 (2013) 10672-10683. 
[24] M.D. Allen, J. Kropat, S. Tottey, J.A. Del Campo, S.S. Merchant, Manganese deficiency in Chlamydomonas results in loss of photosystem II and MnSOD function, sensitivity to peroxides, and secondary phosphorus and iron deficiency, Plant Physiol., 143 (2007) 263277.

[25] O. Osundeko, H. Davies, J.K. Pittman, Oxidative stress-tolerant microalgae strains are highly efficient for biofuel feedstock production on wastewater, Biomass Bioenergy, 56 (2013) 284-294.

[26] O. Osundeko, A.P. Dean, H. Davies, J.K. Pittman, Acclimation of microalgae to wastewater environments involves increased oxidative stress tolerance activity, Plant Cell Physiol., 55 (2014) 1848-1857.

[27] E.H. Harris, The Chlamydomonas Sourcebook, Academic Press, San Diego, 1989. [28] L. Emery, S. Whelan, K.D. Hirschi, J.K. Pittman, Protein phylogenetic analysis of $\mathrm{Ca}^{2+} /$ cation antiporters and insights into their evolution in plants, Front. Plant Sci., 3 (2012) 1. [29] A.K. Bajhaiya, A.P. Dean, L.A.H. Zeef, R.E. Webster, J.K. Pittman, PSR1 is a global transcriptional regulator of phosphorus deficiency responses and carbon storage metabolism in Chlamydomonas reinhardtii, Plant Physiol., 170 (2016) 1216-1234.

[30] D.F. Nathan, M.H. Vos, S. Lindquist, Identification of SSF1, CNS1, and $\mathrm{HCH} 1$ as multicopy suppressors of a Saccharomyces cerevisiae Hsp90 loss-of-function mutation, Proc. Natl. Acad. Sci. U.S.A., 96 (1999) 1409-1414.

[31] C.S. Hassler, K.J. Wilkinson, Failure of the biotic ligand and free-ion activity models to explain zinc bioaccumulation by Chlorella kesslerii, Environ. Toxicol. Chem., 22 (2003) 620626.

[32] J.K. Pittman, N.H. Cheng, T. Shigaki, M. Kunta, K.D. Hirschi, Functional dependence on calcineurin by variants of the Saccharomyces cerevisiae vacuolar $\mathrm{Ca}^{2+} / \mathrm{H}^{+}$exchanger $\mathrm{Vcx} 1 \mathrm{p}$, Mol. Microbiol., 54 (2004) 1104-1116.

[33] J.K. Pittman, C. Edmond, P.A. Sunderland, C.M. Bray, A cation-regulated and proton gradient-dependent cation transporter from Chlamydomonas reinhardtii has a role in calcium and sodium homeostasis, J. Biol. Chem., 284 (2009) 525-533. 
[34] M. Melchionda, J.K. Pittman, R. Mayor, S. Patel, $\mathrm{Ca}^{2+} / \mathrm{H}^{+}$exchange by acidic organelles regulates cell migration in vivo, J. Cell Biol., 212 (2016) 803-813.

[35] R.E. Webster, A.P. Dean, J.K. Pittman, Cadmium exposure and phosphorus limitation increases metal content in the freshwater alga Chlamydomonas reinhardtii, Environ. Sci. Technol., 45 (2011) 7489-7496.

[36] M.G. Palmgren, S. Clemens, L.E. Williams, U. Kraemer, S. Borg, J.K. Schjorring, D. Sanders, Zinc biofortification of cereals: problems and solutions, Trends Plant Sci., 13 (2008) 464-473.

[37] P.K. Menguer, E. Farthing, K.A. Peaston, F.K. Ricachenevsky, J.P. Fett, L.E. Williams, Functional analysis of the rice vacuolar zinc transporter OsMTP1, J. Exp. Bot., 64 (2013) 2871-2883.

[38] S. Eroglu, B. Meier, N. von Wirén, E. Peiter, The vacuolar manganese transporter MTP8 determines tolerance to iron deficiency-induced chlorosis in Arabidopsis, Plant Physiol., 170 (2016) 1030-1045.

[39] Z. Chen, Y. Fujii, N. Yamaji, S. Masuda, Y. Takemoto, T. Kamiya, Y. Yusuyin, K. Iwasaki, S.-i. Kato, M. Maeshima, J.F. Ma, D. Ueno, Mn tolerance in rice is mediated by MTP8.1, a member of the cation diffusion facilitator family, J. Exp. Bot., 64 (2013) 43754387.

[40] C.W. MacDiarmid, M.A. Milanick, D.J. Eide, Induction of the ZRC1 metal tolerance gene in zinc-limited yeast confers resistance to zinc shock, J. Biol. Chem., 278 (2003) 1506515072.

[41] Y. Komine, L.L. Eggink, H.S. Park, J.K. Hoober, Vacuolar granules in Chlamydomonas reinhardtii: polyphosphate and a 70-kDa polypeptide as major components, Planta, 210 (2000) 897-905.

[42] F.A. Ruiz, N. Marchesini, M. Seufferheld, Govindjee, R. Docampo, The polyphosphate bodies of Chlamydomonas reinhardtii possess a proton-pumping pyrophosphatase and are similar to acidocalcisomes, J. Biol. Chem., 276 (2001) 46196-46203. 
[43] N. Das, S. Bhattacharya, M.K. Maiti, Enhanced cadmium accumulation and tolerance in transgenic tobacco overexpressing rice metal tolerance protein gene OsMTP1 is promising for phytoremediation, Plant Physiol. Biochem., 105 (2016) 297-309.

[44] J. Razinger, M. Dermastia, J.D. Koce, A. Zrimec, Oxidative stress in duckweed (Lemna minor L.) caused by short-term cadmium exposure, Environ. Pollut., 153 (2008) 687-694. [45] I. Szivák, R. Behra, L. Sigg, Metal-induced reactive oxygen species production in Chlamydomonas reinhardtii (chlorophyceae), J. Phycol., 45 (2009) 427-435.

[46] K.J. Flynn, A. Mitra, H.C. Greenwell, J. Sui, Monster potential meets potential monster: pros and cons of deploying genetically modified microalgae for biofuels production, Interface Focus, 3 (2013) 20120037. 